American Journal of Pharmacology and Toxicology 1 (4): 83-86, 2006

ISSN 1557-4962

(C) 2006 Science Publications

\title{
Sensitivities of Uterine Adenocarcinoma, Mixed Mullerian Tumor (MMT) and Sarcoma Cell Lines to Chemotherapeutic Agents and a Flex-Het Drug
}

\author{
${ }^{1}$ Johnny Hyde and ${ }^{1,2,3}$ Doris M. Benbrook \\ ${ }^{1}$ Department of Obstetrics and Gynecology and ${ }^{2}$ Department of Biochemistry and Molecular Biology \\ The University of Oklahoma Health Sciences Center, Oklahoma City, OK 73190
}

\begin{abstract}
The administration and combination of a variety of chemotherapeutic agents for treatment of advanced or recurrent uterine cancer of different histologies is under current debate. Mixed Mullerian Tumors (MMTs), which contain both adenocarcinoma and sarcoma components, are the most rate histologic type and it is therefore difficult to conduct clinical trials to determine if they should be treated like endometrial adenocarinomas or like sarcomas. Flexible Heteroarotionoids (FlexHets) are a promising class of anti-cancer drugs with low toxicity that have demonstrated activity against a wide variety of cancer types, but their efficacy in uterine cancers is unknown. The objective of this study was to determine if cell lines established from endometrial carcinoma (HEC-1-A), uterine sarcoma (SK-UT-1) and MMT (MES-SA) cancers exhibit differential sensitivities to cisplatin, carboplatin, paclitaxel, docetaxel, doxorubicin and SHetA2, if SHetA2 can enhance sensitivity to the chemotherapeutic drugs and if SHetA2 exhibits a differential effect on uterine cancer cells in comparison to normal endometrial cells using a cytotoxicity assay. These cell lines did not differ in their sensitivities to platinum or taxel drugs. Doxorubicin was active against the sarcoma but not the adenocarcinoma or MMT cell lines. SHetA2 decreased the survival of all three cell lines, but did not enhance their sensitivities to the chemotherapeutic agents. Two of the three uterine cancer cell lines were more sensitive to SHetA2 in comparison to normal endometrial cells. In conclusion, doxorubicin appears to have a greater effect against sarcoma than other uterine histology types. SHetA2 is affective against uterine cancer cell lines, but does not enhance their sensitivities to chemotherapeutic agents.
\end{abstract}

Key words: Uterine cancer, adenocarcinoma, MMT, sarcoma, doxorubicin, Flex-Het

\section{INTRODUCTION}

Cancer of the uterine corpus is estimated to be detected in 41,200 women per year and to cause 7,350 deaths per year in the United States (www.cancer.org). These deaths are due to cancers that are detected at advanced stage or that recur after primary therapy. Use of chemotherapy drugs for advanced or recurrent uterine cancer is currently being investigated. There are several types of uterine cancer that are being evaluated in clinical trials as separate cancers. About $95 \%$ of uterine cancers are adenocarcinomas, which arise from the endometrial epithelium, while about $2-4 \%$ are sarcomas, which arise from the uterine myometrium. Even more rare, are the uterine carcinosarcomas (also called Mixed Mullerian Tumors or MMTs), which contain both epithelial and myometrial components. There is current debate as to whether MMTs should be treated as adenocarcimas or as sarcomas ${ }^{[1]}$.
The chemotherapeutic agents under investigation in uterine cancer include cisplatin, carboplatin, paclitaxel, docetaxel and doxorubicin. Cisplatin and carboplatin are platinum-containing compounds that cause DNA damage by binding and crosslinking DNA, paclitaxel and docetaxel are diterpenes that interfere with microtubules, doxorubicin is an anthracycline antibiotic that intercalates between DNA base pairs ${ }^{[2]}$. All of these compounds exert significant toxicity in the patients $^{[1-9]}$. In patients with advanced or recurrent endometrial carcinoma, response rates of only $27-54 \%$ have been reported ${ }^{[5-7]}$. Uterine sarcomas and mixed mesodermal tumors are less well studied because of their rarity and have poor response to chemotherapy and poor patient prognosis ${ }^{[3,4]}$.

Flexible-Heteroarotinoids (Flex-Hets) are a novel class of compounds that exhibit promising anti-cancer activity. The lead Flex-Het, called SHetA2, was effective against all cell lines representing 9 different

Corresponding Author: $\quad$ Dr. Doris M. Benbrook, University of Oklahoma Health Sciences Center, 975 NE $10^{\text {th }}$ Street; Room 1372. Oklahoma City, OK 73104, Tel.: 405-271-5523, Fax: 405- 271-3874 
cancer types of the National Cancer Institutes Human Tumor Cell Line Panel in vitro and against an ovarian cancer cell line in $v i v{ }^{[10]}$. Sensitivity of uterine cancers to SHetA2 is currently unknown. Although Flex-Hets were originally modeled after retinoids and retain the retinoid differentiation activity, they do not activate the nuclear retinoid receptors or exhibit the classical retinoid toxicities or teratogenicity ${ }^{[10-13]}$. In addition, Flex-Hets are potent inducers of apoptosis in cancer cells with less effect on normal cells ${ }^{[10,14]}$.

The objective of this study was to determine if cell lines established from endometrial carcinoma (HEC-1A), uterine sarcoma (SK-UT-1) and MMT (MES-SA) cancers exhibit differential sensitivities to cisplatin, carboplatin, paclitaxel, docetaxel, doxorubicin and SHetA2, if SHetA2 can enhance sensitivity to the chemotherapeutic drugs and if SHetA2 exhibits a differential effect on uterine cancer cells in comparison to normal endometrial cells.

\section{MATERIALS AND METHODS}

Cell lines and drugs: All cell lines were obtained from the American Tissue Culture Collection (ATCC). HEC1-A was isolated from a stage IA endometrial cancer, MES-SA was established from a poorly differentiated uterine sarcoma, SK-UT- 1 cells were isolated from a grade III mixed mesodermal tumor (MMT) consistent with leiomyosarcoma. HEC-1-A and MES-SA were cultured in McCoy's 5a medium (modified) with 1.5 $\mathrm{mM}$ L-glutamine adjusted to contain $2.2 \mathrm{~g} \mathrm{~L}^{-1}$ sodium bicarbonate and $10 \%$ fetal bovine serum (FBS). SKUT-1 and primary endometrial cultures were maintained in Minimum Essential Medium (Eagle) (MEM) with $2 \mathrm{mM} \mathrm{L}$-glutamine and Earle's BSS adjusted to contain $1.5 \mathrm{~g} \mathrm{~L}^{-1}$ sodium bicarbonate, 0.1 $\mathrm{mM}$ non-essential amino acids and $1.0 \mathrm{mM}$ sodium pyruvate and $10 \%$ FBS. Primary cultures of normal endometrial cells were isolated from menstrual blood collected from healthy volunteers under and Institutional Review Board (IRB) approved protocol as previously described ${ }^{[15]}$.

Cisplatin, paclitaxel and doxorubicin were obtained from LKT Labs (St. Paul, MN). Carboplatin and docetaxel were obtained from Sigma (St. Louis, MO). The Flex-Het was synthesized and provided by K. Darrell Berlin (Oklahoma State University, Stillwater, $\mathrm{OK})$ as previously described ${ }^{[13]}$. Cisplatin and carboplatin were dissolved in phosphate buffered saline and the others were dissolved in dimethyl sulfoxide (DMSO).
Cytotoxicity assay: Cells were plated at 2000 cells/well in 96 well microtiter plates and allowed to adhere overnight. A separate plate containing 2 fold concentrations of the final drug concentrations was prepared. A multichannel pipetor was used to add $50 \mu \mathrm{l}$ of the $2 \mathrm{X}$ drug solutions to $50 \mu \mathrm{L}$ of the media covering the plated cells. After 3 days of incubation, the media was removed and $50 \mu \mathrm{L}$ each from a plate of $2 \mathrm{X}$ SHetA2 and a plate of $2 \mathrm{X}$ chemotherapeutic drugs were combined in the wells. After an additional 3 days of incubation with the drugs, the percentage of growth inhibition was measured using the CellTiter $96 \mathrm{AQ}_{\text {ueous }}$ Non-Radioactive Cell Proliferation Assay (Promega Corp., Madison, WI), which is composed of a novel tetrazolium compound that is metabolized by viable cells into a soluble formazan that can be quantitated by reading the OD. Each experiment was performed in triplicate and the three values for each treatment were averaged. To calculate survival, the average OD of the treated cultures was divided by that of the control cultures treated with solvent alone for the cisplatin treatments and retinoid alone for the combination treatments.

\section{RESULTS AND DISCUSSION}

Three cell lines, HEC-1-A representing endometrial adenocarinoma, SK-UT-1 representing MMT and MES-SA representing uterine sarcoma were directly compared using a cytotocixity assay for their sensitivities to a variety of chemotherapeutic drugs and SHetA2, which were administered at physiologically achievable concentrations (Fig. 1). All three cell lines were fairly resistant to cisplatin, carboplatin, paclitaxel and docetaxel. Doxorubicin was effective against the uterine sarcoma, but not the adenocarcinoma or MMT. All three cell lines responded to SHetA2 with significantly different sensitivities. The sarcoma was the most sensitive, the MMT was the most resistant and the adenocarcinoma exhibited intermediate sensitivity. The primary endometrial cultures were more resistant to SHetA2 than the adenocarcinoma and sarcoma lines, but not the MMT (Fig. 2). Pre- and post treatment with SHetA2 did not alter the sensitivities of the cell lines to the chemotherapeutic agents (data not shown).

This is the first report directly comparing the chemotherapeutic sensitivities of uterine cancer cell lines representing adenocarcinoma, MMT and sarcoma histologies. These cell lines did not differ in their sensitivities to platinum or taxel drugs. Doxorubicin was active against the sarcoma but not the adenocarcinoma or MMT cell lines. These results address the current debate as to whether MMTs should 


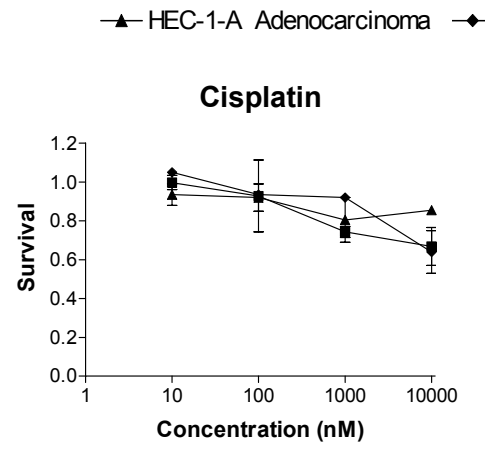

SK-UT-1 MMT - MES-SA Sarcoma
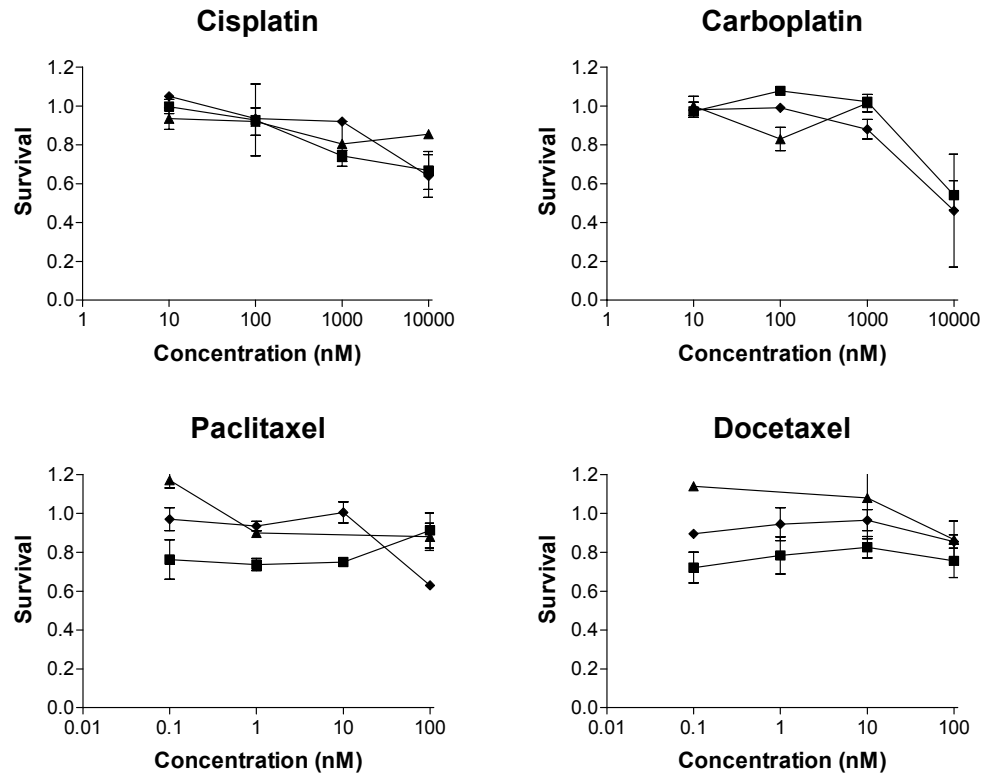

Doxorubicin
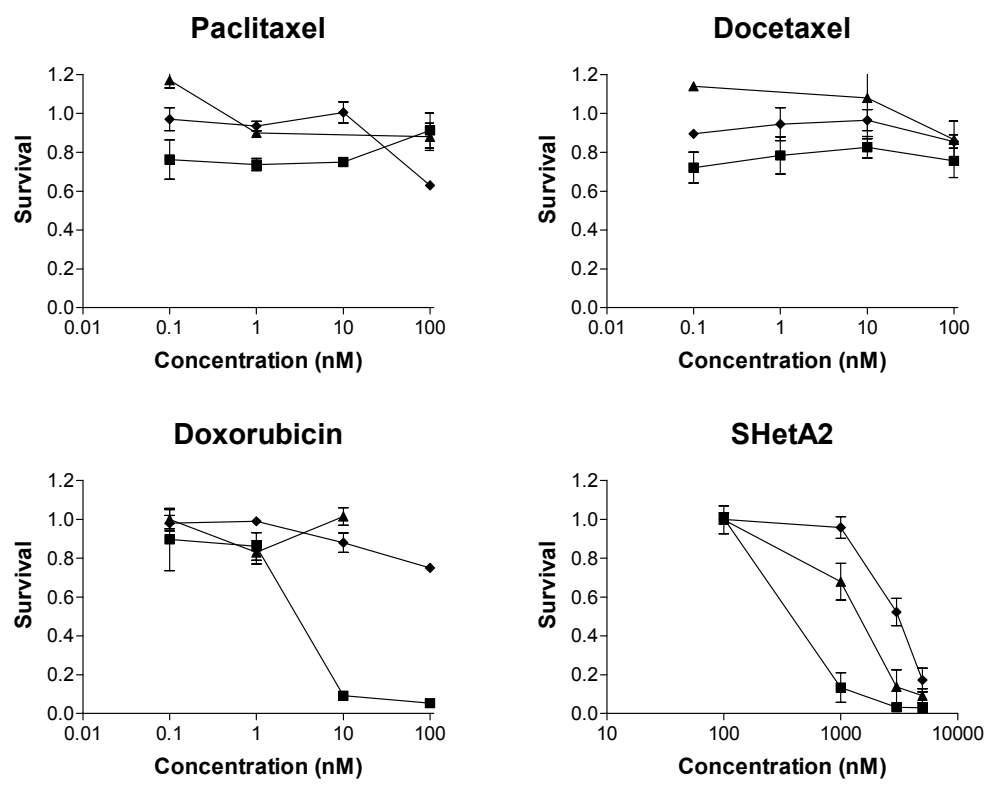

Fig. 1: Effects of Chemotherapeutic Drugs and SHetA2 on Uterine Cancer Cell Lines of Different Histologies. The MTS assay was used to measure survival in cultures treated with a range of physiologically achievable drug concentrations. The $\mathrm{x}$-axis is presented in log scale to allow comparison of the different drugs

SHetA2

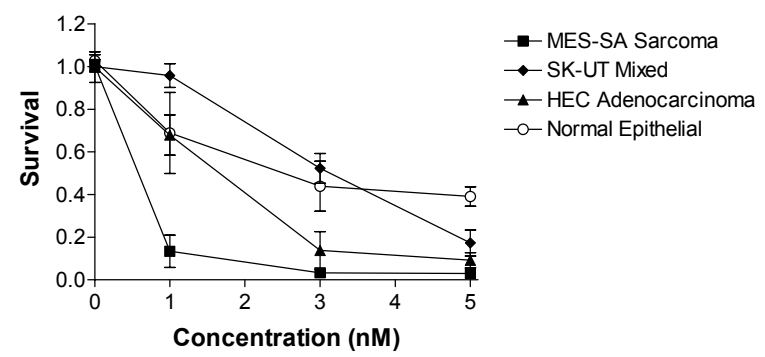

Fig. 2: Differential Effects of HetA2 on Uterine Cancer Cell Lines and Primary Endometrial Cells. The MTS assay was used to measure survival in cultures treated with a range of physiologically achievable drug concentrations. The $\mathrm{x}$-axis is presented in linear scale to highlight the differential cell type sensitivities be treated as adenocarcinomas or sarcomas. The resistance of the adenocarinoma and MMT lines to doxorubicin would support treating patients with MMTs similar to the patients with adenocarinomas. This in vitro study however, suffers from the same limitations as the clinical studies in the rarity of uterine cancer cell lines and patients with MMT and sarcoma histologies. SHetA2 decreased the survival of all three cell lines at physiologically achievable concentrations ${ }^{[16]}$, but did not enhance their sensitivities to the chemotherapeutic agents. Similar to the differential effect of SHetA2 previously observed in ovarian cancer cell lines in comparison to normal and benign cultures ${ }^{[10,13]}$, two of the three uterine cancer cell lines were more sensitive to SHetA2 in comparison to normal endometrial cells. 


\section{CONCLUSION}

Doxorubicin appears to have a greater effect against sarcoma than other uterine histology types. SHetA2 is affective against uterine cancer cell lines, but does not enhance their sensitivities to chemotherapeutic agents.

\section{REFERENCES}

1. McCluggage, W.G., 2002 Uterine carcinosarcomas (malignant mixed Mullerian tumors) are metaplastic carcinomas. Intl. J. Gynecolog. Cancer, 12: 687-90.

2. Falkner, C.A. and D.S. McMeekin, 2005 Staging and Adjuvant Therapy for Endometrial Cancer. The Female Patient, 30: 18-26.

3. O'Meara, A.T., 2004 Uterine sarcomas: have we made any progress? Curr. Opin. Obstet. Gynecol., 16: $1-4$.

4. Dusenbery, K.E., R.A. Potish, P.A. Argenta and P.L. Judson, 2005 On the apparent failure of adjuvant pelvic radiotherapy to improve survival for women with uterine sarcomas confined to the uterus. Am. J. Clin. Oncol., 28: 295-300.

5. Fleming, G.F., V.L. Brunetto, D. Cella, K.Y. Look, G.C. Reid, A.R. Munkarah, R. Kline, R.A. Burger, A. Goodman and R.T. Burks, 2004 Phase III trial of doxorubicin plus cisplatin with or without paclitaxel plus filgrastim in advanced endometrial carcinoma: A Gynecologic Oncology Group Study. J. Clin. Oncol., 22: 2159-66.

6. Fleming, G.F., V.L. Filiaci, R.C. Bentley, T. Herzog, J. Sorosky, L. Vaccarello and H. Gallion, 2004 Phase III randomized trial of doxorubicin + cisplatin versus doxorubicin $+24-\mathrm{h}$ paclitaxel + filgrastim in endometrial carcinoma: A Gynecologic Oncology Group study [see comment]. Ann. Oncol., 15: 1173-8.

7. Thigpen, J.T., M.F. Brady, H.D. Homesley, J. Malfetano, B. DuBeshter, R.A. Burger and S. Liao, 2004 Phase III trial of doxorubicin with or without cisplatin in advanced endometrial carcinoma: A Gynecologic Oncology Group Study. J. Clin. Oncol., 22: 3902-8.

8. Gourley, C., A. Al-Nafussi, M. Abdulkader, J.F. Smyth and H. Gabra, 2002 Malignant mixed mesodermal tumours: biology and clinical aspects. Eur. J. Cancer, 38: 1437-46.
9. Kanjeekal, S., A. Chambers, M.F.K. Fung and S. Verma, 2005 Systemic therapy for advanced uterine sarcoma: A systematic review of the literature. Gynecolog. Oncol., 97: 624-37.

10. Benbrook, D.M., S.A. Kamelle, S.B. Guruswamy, S.A. Lightfoot, B. Hannafon, T.L. Rutledge, N.S. Gould, S.T. Dunn and K.D. Berlin, 2005 Flexible heteroarotinoids (Flex-Hets) exhibit improved therapeutic ratios as anti-cancer agents over retinoic acid receptor antagonists. Inv. New Drugs, 23: 417-428.

11. Guruswamy, S., S. Lightfoot, M. Gold, R. Hassan, K.D. Berlin, R.T. Ivey and D.M. Benbrook, 2001 Effects of retinoids on cancerous phenotype and apoptosis in organotypic culture of ovarian carcinoma. J. Nat. Cancer Inst., 93: 516-525.

12. Mic, F.A., A. Molotkov, D.M. Benbrook and G. Duester, 2003 Retinoid activation of RAR but not RXR is sufficient for mouse embryonic development. Proc. Natl. Acad. Sci., 100: 71357140.

13. Liu, S., C.W. Brown, K.D. Berlin, A. Dhar, S.B. Guruswamy, D. Brown, G.J. Gardner, M.J. Birrer and D.M. Benbrook, 2004 Synthesis of flexible sulfur-containing heteroarotinoids that induce apoptosis and reactive oxygen species with discrimination between malignant and benign cells. J. Med. Chem., 47: 999-1007.

14. Chun, K.-H., D.M. Benbrook, K.D. Berlin, W.K. Hong and R. Lotan, 2003. Induction of apoptosis in head and neck squamous cell carcinoma (HNSCC) cell lines by heteroarotinoids through a mitochondrial dependent pathway. Cancer Res., 63: 3826-3832.

15. Kamelle, S., A. Sienko and D.M. Benbrook, 2002 Retinoids and steroids regulate menstrual phase histological features in human endometrial organotypic cultures. Fertility and Sterility, 78: 596-602.

16. Zhang, Y., Y. Hua, D.M. Benbrook, J.M. Covey and K.K. Chan, 2006. High performance liquid chromatographic analysis and preclinical pharmacokinetics of the heteroarotinoid antitumor agent, SHetA2. Cancer Chemother. Pharmacol., (In press). 